\title{
INHALTSVERZEICHNIS ZUM TAFELBAND
}

ANELL, BenGt

Flügelnetze in der Südsee. . . . . . . . . . . . . . . . . . . . . . . Tf. 1- 2 BODROGI, TIBOR

Kapkap in Melanesien . . . . . . . . . . . . . . . . . . . . . . . Tf. 3-14

BÖTTGER, WALTER

Die Leipziger Emma-ō-Plastik und ihre Restaurierung . . . . . . . . . . Tf. 15- 20

DRÄGER, LOTHAR

Ein bemaltes Tipi der Dakota-Indianer im Museum für Völkerkunde Leipzig Tf. $21-22$

Drost, Dietrich

Eine Reiterdarstellung aus dem Kameruner Grasland . . . . . . . . . . Tf. 23- 26

Finsterbusch, Käte

Die Mundorgeln des Museums für Völkerkunde zu Leipzig und ihre Darstellung

in Ost- und Südostasien . . . . . . . . . . . . . . . . . . . . . . Tf. 27-44

Fischer, HaNS

Spiele der Wotut (Ost-Neuguinea) . . . . . . . . . . . . . . . . . Tf. 45-50

GERMER, ERNST

Miklucho-Maklai und die koloniale Annexion Neuguineas durch das kaiser-

liche Deutschland . . . . . . . . . . . . . . . . . Tf. 51-54

HaBerland, Eike

Eisen und Schmiede in Nordost-Afrika . . . . . . . . . . . . Tf. 55- 56

HoFFMANN, EDITH

Zur Geschichte der vorgeschichtlichen Sammlung des Museums für Völker-

kunde in Leipzig . . . . . . . . . . . . . . . . . . . Tf. 57-60

HöLTKER, GEORG

Leichenbrand und anderes vom unteren Ramu (Neuguinea) . . . . . . . . Tf. 61-64

ISRAEL, HEINZ

Bemerkungen zu einigen verzierten Walroßzähnen aus Südwest-Alaska . . Tf. $65-70$

KONRAD, WALTER

Die „Kanaken" des Tschadsees . . . . . . . . . . . . . . . . . . Tf. $71-72$

KRIEGER, KURT

Töpferei der Hausa . . . . . . . . . . . . . . . . . . . . . . . . . Tf. $73-80$

Kunz, Ludvík

Fenich und Waldkorn . . . . . . . . . . . . . . . . . . . . Tf. 81-84

LaRSSON, KarL ERIK

Ein figürlicher Aufhängehaken . . . . . . . . . . . . . . . . Tf. $85-86$

LIPS, Eva

Ethnobotanisches zum „Zuckerahorn“ . . . . . . . . . . . . . . . . . Tf. 87-94

LOMMEL, ANDREAS

Stilistische Vergleiche an australischen Felsbildern . . . . . . . . . . . . Tf. 95-102

Menzel, BrigitTe

Wechselausstellungen im Museum für Völkerkunde Berlin . . . . . . . . Tf. 103-104

MoschKaU, RUdolF

Zwei vorgeschichtliche Felsbilder an den Thomassteinen im Zittauer Gebirge Tf. 105-106

Neumann, Peter

Eine verzierte Kalebassenschüssel aus Suriname . . . . . . . . . . . . Tf. 107-110 
SChLENTHER, URSULA

Eine argentinische archäologische Steinsammlung im Institut für Ur- und

Frühgeschichte der Humboldt-Universität zu Berlin . . . . . . . . . . . Tf. 111-122

SChUBERT, JoHANnes

Aus Nordindien und dem Himālaya. Notizen zu einigen für das Museum für

Völkerkunde Leipzig erworbenen Gegenständen . . . . . . . . . . . . Tf. 123-128

SiEvers, LORE

Das Herrnhuter Völkerkundemuseum ． . . . . . . . . . . . . . . . . Tf. 129-132

Spannaus, Günther

Das Häuptlingswesen der Ndau in Südostafrika . . . . . . . . . . . . . Tf. 133-134

Termer, Franz

Der erste Bericht über die Ruinen von Uxmal, Yucatan, aus dem 16. Jahr-

hundert . . . . . . . . . . . . . . . Tf. 135-138

TISCHNER, HERBERT

Beiträge zur Ethnographie des alten Viti Levu und Vanua Levu nach unver-

öffentlichten Notizen und Zeichnungen Theodor Kleinschmidts aus den

Jahren 1875-78 . . . . . . . . . . . . . . . . . . . . . Tf. 139-146

WestPhal-Hellbusch, S.

Der Reisanbau im südlichen Iraq . . . . . . . . . . . . . . . . . . Tf. 147-150

WOLF, SIEGFried

Zwei Benin-Arbeiten im Staatlichen Museum für Völkerkunde Dresden: Vogel-

gestaltiges Zeremonialgerät und Reliefplatte mit Vogel

Tf. $151-152$ 